\title{
Endoscopic Posterior Cricoid Split with Costal Cartilage Graft: A Fifteen-Year Experience
}

John P. Dahl, MD, PhD, MBA1,2,*, Patricia L. Purcell, MD¹, MPH, Sanjay R. Parikh, MD, FACS $^{1}$, and Andrew F. Inglis Jr, MD ${ }^{1}$

Running Title: EPCS/RG: A Fifteen-Year Experience

${ }^{1}$ Seattle Children's Hospital, Department of Otolaryngology: Head \& Neck Surgery, University of Washington, Seattle, WA 98145, USA

${ }^{2}$ Indiana University School of Medicine, Department of Otolaryngology-Head \& Neck Surgery, Indianapolis, IN USA

Financial Disclosure: None

Conflict of Interest: None

Presented at the American Society of Pediatric Otolaryngology Spring Meeting, Boston, MA, USA, April 23-26, 2015

*Corresponding Author: John P Dahl, MD, PhD, MBA, Indiana University School of Medicine, Department of Otolaryngology-Head and Neck Surgery, 1120 W Michigan Street, Gatch Hall, Suite 200, Indianapolis, IN 46202-5209. jpdahl@iu.edu. (317) 274-1259 Fax (317) 274-8285

This is the author's manuscript of the article published in final edited form as:

Dahl, J. P., Purcell, P. L., Parikh, S. R., \& Inglis, A. F. (2017). Endoscopic posterior cricoid split with costal cartilage graft: A fifteen-year experience. The Laryngoscope, 127(1), 252-257. https://doi.org/10.1002/lary.26200 


\begin{abstract}
Objective: To evaluate outcomes of the endoscopic posterior cricoid split with rib graft (EPCS/RG) procedure in the treatment of subglottic stenosis (SGS), posterior glottic stenosis (PGS), and bilateral vocal fold immobility (BVFI).

Study Design: Retrospective chart review.

Methods: Chart review of all patients who underwent EPCS/RG at a single tertiary care facility between 1999 and 2014. Patients were grouped based on the primary indication for the procedure. Decannulation was the primary endpoint. Secondary endpoints were the number of subsequent airway procedures and length of hospitalization.
\end{abstract}

Results: 33 patients were identified; 32 had tracheotomy. Overall decannulation rate was $65.6 \%$. Subgroup analysis demonstrated the following decannulation rates: $53.8 \%$ for SGS, $100 \%$ for PGS, and 28.6\% for BVFI. Fisher Exact Test found a significant difference in overall decannulation rates between groups $(\mathrm{p}=0.002)$. Operation-specific decannulation rates for patients who never required an open procedure were 23\% for SGS, 91.6\% for PGS and 28.6\% for BVFI. This difference was also statistically significant $(\mathrm{p}=0.001)$. Multivariate logistic regression analysis found that prematurity had a positive correlation with decannulation that approached statistical significance ( $\mathrm{p}<0.051$, OR 6.1, 95\% CI 0.99, 37.6). The percentage of patients who underwent repeat airway procedures for the groups was $61.5 \%$ for SGS, $16.6 \%$ for PGS, and $14.3 \%$ for BVFI. The median length of hospitalization after EPCS/RG was 3 days.

Conclusion: This represents the largest series of patients who have undergone EPCS/RG and demonstrates that the majority of patients can be decannulated after this procedure. Patients with PGS had the highest operation-specific decannulation rates.

Keywords: endoscopic posterior cricoid split with rib graft, subglottic stenosis, posterior glottic stenosis, and bilateral vocal fold immobility

Level of Evidence: 4 
Introduction:

Despite advances in open and endoscopic airway surgery, subglottic stenosis (SGS), posterior glottic stenosis (PGS), and bilateral vocal fold immobility (BVFI) continue to present a significant challenge to airway surgeons. Patients with these conditions often undergo multiple surgical procedures, and are often tracheotomy dependent for extended periods of time.

The endoscopic posterior cricoid split with rib grafting (EPCS/RG) procedure was developed in the late 1990's as a less invasive surgical approach for the treatment of these conditions. The initial case series of 10 patients, including description of the procedure, was published in $2003 .{ }^{1}$ Since that time, other surgeons have reported their experiences and outcomes with the procedure. The present study aims to examine our 15-year experience with EPCS/RG via a retrospective review and expand upon the available clinical outcomes data for children who have undergone the procedure.

Materials and Methods:

After obtaining Institutional Review Board approval, we identified all patients who underwent airway reconstruction for SGS, PGS, or BVFI using an autologous cartilage graft from January 1, 1999, to October 6, 2014, at Seattle Children's 
Hospital. The EPCS/RG procedure was performed as previously described (Figure 1). ${ }^{1}$

Subjects were grouped based on the indication for the EPCS/RG procedure: SGS, PGS, or BVFI. No patients were excluded for concomitant diagnoses. For each patient that had complex laryngeal stenosis, close review of clinical and operative reports allowed identification of the primary diagnosis so that patients could be categorized accordingly. All patients with PGS also had arytenoid ankyloses (AA) to some degree; therefore these diagnoses were categorized together, as per the Bogdasarian Olson classification system ${ }^{2}$. The diagnosis of PGS was made in patients with a history of intubation trauma. The diagnosis of bilateral VF immobility was based on endoscopic evaluation, including palpation.

In addition to the indication for surgery, we collected demographic characteristics regarding medical comorbidities, age at time of surgery, and whether or not a child was premature. Decannulation rates were considered the primary endpoint in our analysis; therefore, children who did not undergo tracheotomy were excluded from the final analysis. In addition, some children had medical comorbidities that may have prevented decannulation. We included these children in our overall analysis to reflect the true practical outcome of the procedure; however, we did perform a secondary analysis of decannulation rates in which they were excluded.

Analysis 
Descriptive statistical analyses were used to evaluate the demographic characteristics of the patient population. Analysis of variance (ANOVA) and Fisher Exact Test were utilized to determine if there were any differences in baseline characteristic among the groups as well as to determine if there were any differences in decannulation rates. Multivariate logistic regression analyses were then utilized to determine whether indication for surgery, prematurity, or age at the time of surgery impacted decannulation rates. Additional comparisons were made between the PGS and SGS groups using Fisher Exact Test due to rarity of outcomes.

$P<0.05$ was considered statistically significant. Stata 13.1 (Stata Inc, College Station, TX) statistical software was used for all analyses.

Results:

A total of 33 patients who underwent EPCS/RG were identified; one was excluded as the patient never underwent tracheotomy. Therefore, 32 patients were included in the final analysis. There were 13 patients with SGS, 12 with PGS and 7 with BVFI. There were 11 patients with some degree of both PGS/AA \& SGS; final categorization was dependent upon which level of obstruction was the most severe. One patient had both BVFI and PGS; this patient was categorized with the BVFI group.

Twenty-one patients were eventually decannulated, for an overall decannulation rate of $65.6 \%$. Five patients had medical comorbidities potentially preventing 
decannulation. One patient with BVFI required pulmonary toilet due to encephalopathy of prematurity. Four patients with SGS had the following comorbidities: Crouzon syndrome, severe recurrent respiratory papillomatosis, cervical lymphatic malformation and static encephalopathy. One patient with BVFI was lost to follow-up. If we exclude the patients with medical co-morbidities that prohibited their decannulation or who were lost to follow-up, the overall decannulation rate was $80.8 \%$.

Among all 32 patients, there were 16 who underwent EPCS/RG and were decannulated without requiring open procedure. If operation-specific refers to avoidance of any open procedure, then the operation-specific decannulation rate for endoscopic surgery alone was 50\%. If we exclude the 6 patients with comorbidities preventing decannulation or who were lost to follow-up, then the operation-specific decannulation rate of EPCS/RG increased to 61.5\%.

The demographic characteristics of the patient population are outlined in Table 1. There were no significant differences in age at the time of surgery among the SGS, PGS, and BVFI groups. ANOVA analysis found a significant difference between the BVFI group and the other two groups in terms of the proportion who were premature $(\mathrm{p}<0.046)$.

Among the 32 patients, the overall decannulation rates for the groups are: $53.8 \%$ for the SGS group, $100 \%$ for the PGS group, and $28.6 \%$ for the BVFI group (Figure 2). 
Fisher Exact Test found a significant difference in overall decannulation rates between groups $(\mathrm{p}=0.002)$. Operation-specific decannulation rates for patients who never required an open procedure were as follows: $23 \%$ for SGS, $91.6 \%$ for PGS and 28.6\% for BVFI (Figure 3). Fisher Exact Test also found a significant difference in operation-specific decannulation rates between groups, $(\mathrm{p}=0.001)$.

When rating the severity of stenosis, we used the Bogdasarian Olson classification ${ }^{2}$ for children with PGS. Retrospective review identified 2 children with Grade III PGS and 11 children with Grade IV PGS, one of whom was excluded for lack of tracheotomy. For children with SGS, we used the Cotton-Myer classification ${ }^{3}$ system, identifying 4 children with Grade II SGS and 9 children with Grade III SGS.

The children with Grade III PGS had an operation-specific decannulation rate of $100 \%$, the rate decreased slightly to $90 \%$ for children with Grade IV PGS. Among the children with SGS, 50\% with Grade II SGS were successfully decannulated without an open procedure. The operation-specific decannulation rate for Grade III SGS was $17 \%$.

Most children (21/32,65.6\%) did not undergo a subsequent endoscopic or open graft procedure. Five children (15.6\%) underwent revision EPCS/RG; one of these children also underwent a third EPCS/RG. Seven children (21.8\%) underwent open graft procedure. Only one child underwent both a revision endoscopic and an open graft procedure. 
The children with SGS had higher rates of revision airway procedures, both endoscopic and open. Five patients with SGS (38.5\%) required a subsequent open airway surgery, $2(15.4 \%)$ required a second EPCS/RG procedure, and $1(7.7 \%)$ required both (Table 1). In the PGS Group, 1 patient (8.3\%) required an open airway surgery and $1(8.3 \%)$ patient required a second EPCS/RG procedure (Table 1). Only one patient (14.3\%) in the BFVI group underwent a second airway reconstruction, an EPCS/RG procedure. Of note, 6 patients in the PGS group underwent tracheotomy at the time of their EPCS/RG procedure; all 6 were successfully decannulated.

A multivariate logistic regression analysis that controlled for indication for surgery found that prematurity had a positive correlation with decannulation that approached significance $(\mathrm{p}<0.051, \mathrm{OR} 6.1,95 \% \mathrm{CI} 0.99,37.6)$. The median length of hospitalization after EPCS/RG was 3 days. The median length of follow-up for the entire group was 3 years (range 0 - 15 years). Median follow-up time was 2 years for patients not decannulated and 3 years for patients who were decannulated.

In addition, we identified factors that may be associated with successful operationspecific decannulation among the 32 patients. Of 21 patients with pulmonary disease, only $38 \%$ were successfully decannulated without open procedure. The decannulation rate increased to $72.7 \%$ among the 11 patients without pulmonary disease. However, the difference was not significant, $p=0.14$, likely due to 
inadequate power. There were too few patients with major cardiac comorbidities to evaluate the effect of this condition on decannulation outcomes.

We also found differences in operation-specific decannulation rates among patients with pre-existing tracheotomies to those who underwent tracheotomy at the time of surgery. Six patients with PGS underwent tracheotomy at time of EPCS/RG. All 6 of these patients were decannulated shortly after their surgery, while only 10 of the 26 with pre-existing tracheotomy were decannulated. The difference was significant with Fisher Exact Test, $\mathrm{p}=0.002$.

There were too few patients with Grade II SGS and Grade III PGS to allow for statistical comparison of decannulation rates based on grade of stenosis. However, there was a significant difference in operation-specific decannulation rates between patients with PGS and SGS. Of the 12 patients with PGS, 11 (92\%) were decannulated without requiring open procedure. In contrast, 3 (23\%) of the 13 patients with SGS were decannulated without open procedure, $p=<0.001$.

There were 5 patients with pre- and post-operative videofluoroscopic swallow studies (VFSS). Of these, 3 developed worsening dysphagia after surgery. Twentytwo of the patients had a G-tube during the perioperative period.

In our series of 7 patients with BVFI, 2 were decannulated, 1 was lost to follow-up, and 1 could not be decannulated due to need for pulmonary toilet. The remaining 3 
patients had persistent symptoms associated with airway obstruction, but they also had some preservation of voice.

Discussion:

We originally described the EPCS/RG procedure in 2003. Since the initial publication, surgeons have been able to reproduce the results with this or similar approaches in the treatment of SGS, PGS, and BVFI. In 2004, Rutter and Cotton demonstrated a $97 \%$ decannulation rate in a population of 29 patients with PGS or BVFI using an open approach to place a costal cartilage graft. ${ }^{4}$ Despite an excellent overall decannulation rate, this procedure requires at the minimum a partial laryngofissure, and the majority of the patients in the series required a full laryngofissure. Using EPCS/RG, Provenzano et al. (2011) demonstrated a 67\% decannulation rate in a series of 12 patients and was the largest population of such patients reported in the literature at that time. ${ }^{5}$ Gerber et al. (2013) subsequently published a series of 28 patients who underwent EPCS/RG procedures at 3 tertiary care centers with $89 \%$ of patients either avoiding a tracheotomy or being successfully decannulated. ${ }^{6}$

The primary goal of this study was to examine the surgical outcomes of the EPCS/RG procedure in the treatment of SGS, PGS, and BFVI. ${ }^{1}$ As described in our initial report, patients undergoing EPCS/RG at our institution also undergo tracheotomy at the time of EPCS/RG if one is not present pre-operatively. The senior author of this 
study feels that a tracheotomy should be in place at the time of EPCS/RG, as dislodgement of the costal cartilage graft into the distal airway would cause significant airway obstruction and possibly death. We therefore chose decannulation as the primary endpoint for this study, while also considering other endpoints such as the need for additional airway procedures.

In this study, only 6 patients underwent perioperative tracheotomy, and all had PGS. When counseling families regarding the options for endoscopic versus open pediatric airway surgery, we consider several factors, which have been summarized in Figure 4 as a proposed treatment algorithm.

In the patient with chronic airway obstruction, we consider both the site of the lesion and whether or not the patient is tracheotomy dependent (Figure 4). If the patient is tracheotomy dependent with solely PGS or with Grade 3 or better posteriorly based SGS, then we are more likely to recommend EPCS/RG as first-line therapy. The vast majority of children in this series had a pre-existing tracheotomy; proceeding with endoscopic surgery in this circumstance is arguably less invasive than an open procedure. If the patient is tracheotomy dependent, but has Grade IV SGS, multiple sites of obstruction, and/or a significant anterior stenosis, then we recommend an open procedure to allow placement of anterior or both anterior and posterior grafts. 
For children who do not have a tracheotomy and who only require a posterior graft due to PGS, we discuss with families the options of 1) endoscopic surgery with a perioperative tracheotomy versus 2) open single stage posterior graft with approximately weeklong period of intubation with sedation in the ICU. There are significant risks that must be considered in either case. For families who desire for their child to be able to participate in therapies more quickly, endoscopic surgery with a tracheotomy may indeed be preferable, particularly considering that almost all children in this series were decannulated within several weeks of surgery. However, some families may feel strongly opposed to tracheotomy for any period of time, in which case we may proceed with the open procedure. If the patient is not tracheotomy dependent and has SGS or needs an anterior graft, then we would recommend open procedure.

In addition, 3 patients in this series developed worsening dysphagia after surgery. If patients are feeding orally preoperatively, we believe it is important to counsel families that swallowing may worsen after surgery.

The overall decannulation rate for this series of 32 patients who underwent EPCS/RG was found to be $65.6 \%$. This is comparable to the $67 \%$ decannulation rate reported by Provenzano et al (2011) in their series of 12 patients who underwent EPCS/RG. ${ }^{5}$ However, if we exclude patients lost to follow-up or with medical comorbidities that precluded their decannulation, the overall decannulation rate for this series rises to $80.8 \%$. 
We further examined our decannulation rates based on the indication for EPCS/RG and found a statistically significant difference in the decannulation rates among the three indications for EPCS/RG. Patients with PGS had the highest decannulation rates. As stated above, 6 patients in the PGS group underwent tracheotomy at the time of their EPCS/RG procedure, and all were subsequently decannulated. The fact that these patients were not tracheotomy dependent preoperatively may be one reason the patients in the PGS group had such a high decannulation rate compared to those undergoing EPCS/RG for SGS or BVFI. We also found that children with SGS were more likely to require a subsequent open procedure. Overall, we found that EPCS/RG may be of most benefit in treating posteriorly based lesions at the level of the glottis; therefore it may be particularly useful for treating isolated PGS.

Interestingly, Thakkar and Gerber published an initial series of 2 cases of EPCS/RG for BVFI, neither of which underwent tracheotomy, and argued that the EPCS/RG procedure is an alternative to tracheotomy for BVFI.7 A subsequent, multiinstitutional case series of 28 patients who underwent EPCS/RG included 9 patients with a diagnosis of BVFI. ${ }^{6}$ Six of the patients with BVFI had a tracheotomy in place prior to the EPCS/RG procedure or during the perioperative period and all 6 were subsequently decannulated. The remaining 3 patients never underwent tracheotomy. ${ }^{6}$ In our series of 7 patients with BVFI, 2 were decannulated, 1 was lost to follow-up, and 1 could not be decannulated due to need for pulmonary toilet. The remaining 3 patients had persistent symptoms associated with airway obstruction. 
While they remained tracheotomy dependent, they also continued to have some ability to voice, which was important to them. It is possible that additional procedures could have improved their airway, but further compromised their voice. The difference in decannulation rates for patients who have undergone EPCS/RG for BVFI between the two studies may represent variations in decannulation protocols at the institutions involved with these studies, as the procedures appear to be performed in a similar way by all of the surgeons. Rate of decannulation is not a perfect outcome measure as providers may have different preferences regarding when and how strongly to pursue decannulation.

When comparing the results of this study with the outcomes reported by Gerber et al, we find similar rates of decannulation for patients with PGS. In both cases, all children with PGS were successfully decannulated; however, the Gerber et al (2013) study only reported 3 patients with PGS. ${ }^{5}$ In addition, both studies demonstrated that patients with SGS who underwent EPCS/RG were more likely to require other airway procedures prior to decannulation. In the Gerber et al (2013) series, 12 of the 14 SGS patients who had a tracheotomy at the time of their EPCS/RG procedure were decannulated, 5 of whom required additional airway surgeries in order to achieve decannulation. ${ }^{5}$ This is not unexpected given that the airway obstruction seen in patients with SGS is usually not limited to the posterior subglottis and is thus less likely to be corrected by expanding the posterior airway via an EPCS/RG procedure. 
We acknowledge that it can be difficult to make outcome comparisons in pediatric airway surgery for several reasons. First, we are performing the EPCS/RG procedure among a select group of patients who we anticipate will benefit from a posterior graft alone. Perhaps the best method to determine the utility of EPCS/RG would be a randomized trial of EPCS/RG versus open single-stage posterior graft. However, such a study is not likely to be feasible due to the rarity of the condition. Expansion laryngoplasty and resection techniques can be done for a wide variety of airway lesions, including patients who we would not consider candidates for EPCS/RG. Second, decannulation rate as an outcome measure does not fully represent the benefit patients receive from the EPCS/RG procedure. Ideally, a study would include objective pre- and post-operative measures of activity tolerance, sleep apnea and feeding/swallowing. As this is a retrospective case series, we are limited by the data that is available in the medical record. Finally, if we limit our analysis to only patients with PGS, we calculate an operation-specific decannulation rate of $92 \%$ using EPCS/RG alone. The operation-specific decannulation rate decreases when we consider other airway lesions. Differences in decannulation rates could be due to differences in institutional preferences regarding which lesions to treat endoscopically.

One advantage of the EPCS/RG procedure is a decreased duration of hospitalization and lower intensive care unit utilization, unless the patient undergoes tracheotomy at the time of surgery. The present study found a median length of stay of 3 days, which is comparable to our previous findings. ${ }^{1}$ Provenzano et al (2011) reported 
an average length of stay of 3.6 days while Gerber et al (2013) did not report any data regarding duration of hospitalization. Of note, there are 6 reported cases in which EPCS/RG was performed without tracheotomy. If this is confirmed to be safe in subsequent studies, it may be possible to avoid the costs and risks associated with discharging children with tracheotomy tubes from the hospital.

The obvious limitation of the present study is the retrospective nature in which outcome data were collected. Ideally, such data would have been collected in a prospective manner along with a detailed record of surgical complications, vocal outcomes, as well as swallowing outcomes. As we continue to study the outcomes from EPCS/RG, it will also be important to examine intensive care unit and home tracheotomy care resource utilization compared to other airway reconstruction techniques.

It may also be of interest to compare technical details of the procedure among surgeons with experience performing EPCS/RG in order to determine the impact of technical refinements in the procedure on surgical outcomes. More research should also be conducted into the role for revision EPCS/RG. We consider the approach for revision EPCS/RG to be similar to primary surgery; however, the most notable difference is that the characteristics of the cricoid often appear different due to partial or total graft resorption. The revision graft must be carefully carved as the anterior and posterior flanges may be more difficult to fit appropriately. It is 
possible that such challenges may impact overall outcomes, and thus further investigation is needed into outcomes of revision EPCS/RG.

Conclusions:

EPCS/RG represents a safe and effective surgical approach, particularly for children with isolated PGS. We identified the highest overall and operation-specific decannulation rates among children with PGS, although children with SGS and BVFI were also successfully decannulated without open surgery. Children with SGS were more likely to require subsequent open procedure. Voice outcomes may limit decannulation rates for some patients with BVFI. This study represents our single institutional series of patients who underwent EPCS/RG and demonstrates that the majority of patients undergoing this procedure are successfully decannulated. 
References:

1. Inglis AF, Jr., Perkins JA, Manning SC, Mouzakes J. Endoscopic posterior cricoid split and rib grafting in 10 children. The Laryngoscope 2003; 113:2004-2009.

2. Bogdasarian RS, Olson NR. Posterior glottis laryngeal stenosis. Otolaryngol Head Neck Surg (1979). 1980; 88(6): 765-72.

3. Myer CM 3 $3^{\text {rd }}$ O’Connor DM, Cotton RT. Proposed grading system for subglottic stenosis based on endotracheal tube sizes. Ann Otol Rhinol Laryngol. 1994; 103(4 Pt 1): 319-23.

4. Rutter MJ, Cotton RT. The use of posterior cricoid grafting in managing isolated posterior glottic stenosis in children. Archives of otolaryngology--head \& neck surgery $2004 ; 130: 737-739$.

5. Gerber ME, Modi VK, Ward RF, Gower VM, Thomsen J. Endoscopic posterior cricoid split and costal cartilage graft placement in children. Otolaryngology--head and neck surgery : official journal of American Academy of Otolaryngology-Head and Neck Surgery 2013; 148:494-502. 
6. Provenzano MJ, Hulstein SL, Solomon DHet al. Pediatric endoscopic airway management with posterior cricoid rib grafting. The Laryngoscope $2011 ; 121: 1062-$ 1066.

7. Thakkar K, Gerber ME. Endoscopic posterior costal cartilage graft placement for acute management of pediatric bilateral vocal fold paralysis without tracheostomy. International journal of pediatric otorhinolaryngology 2008; 72:1555-1558. 
Table 1: Demographic and Outcome Data

\begin{tabular}{|c|c|c|c|c|c|c|c|}
\hline Indication & $\begin{array}{l}\text { Average } \\
\text { Age at } \\
\text { Surgery }\end{array}$ & Premature & $\begin{array}{c}\text { Decannulated } \\
\text { without open } \\
\text { procedure }^{\#}\end{array}$ & $\begin{array}{c}\text { Decannulated } \\
\text { Overall }\end{array}$ & $\begin{array}{l}\text { Requiring } \\
\text { Open } \\
\text { Procedure }\end{array}$ & $\begin{array}{l}\text { Requiring } \\
\text { Second } \\
\text { EPCS/RG } \\
\text { Procedure }\end{array}$ & $\begin{array}{c}\text { Requiring both an } \\
\text { Open and Repeat } \\
\text { EPCS/RG } \\
\text { Procedure }\end{array}$ \\
\hline $\begin{array}{l}\text { Subglottic } \\
\text { Stenosis } \\
(n=13)\end{array}$ & $\begin{array}{c}4.15 \\
\text { years }\end{array}$ & $\begin{array}{c}n=10 \\
(76.9 \%)\end{array}$ & $\begin{array}{c}n=3 \\
(23 \%)\end{array}$ & $\begin{array}{c}n=7 \\
(53.8 \%)\end{array}$ & $\begin{array}{c}n=5 \\
(38.5 \%)\end{array}$ & $\begin{array}{c}n=2 \\
(15.4 \%)\end{array}$ & $\begin{array}{c}n=1 \\
(7.7 \%)\end{array}$ \\
\hline $\begin{array}{l}\text { PGS } \\
(n=12)\end{array}$ & 8 years & $\begin{array}{c}n=10 \\
(83.3 \%)\end{array}$ & $\begin{array}{c}n=11 \\
(91.7 \%)\end{array}$ & $\begin{array}{c}n=12 \\
(100 \%)\end{array}$ & $\begin{array}{c}n=1 \\
(8.3 \%)\end{array}$ & $\begin{array}{c}n=1 \\
(8.3 \%)\end{array}$ & $\begin{array}{l}n=0 \\
(0 \%)\end{array}$ \\
\hline $\begin{array}{l}\text { BVFI } \\
(n=7)\end{array}$ & $\begin{array}{c}3.3 \\
\text { years }\end{array}$ & $\begin{array}{c}n=2^{*} \\
(28.6 \%)\end{array}$ & $\begin{array}{c}n=2 \\
(28.6 \%)\end{array}$ & $\begin{array}{c}n=2 \\
(28.6 \%)\end{array}$ & $\begin{array}{l}n=0 \\
(0 \%)\end{array}$ & $\begin{array}{c}n=1 \\
(14.3 \%)\end{array}$ & $\begin{array}{l}n=0 \\
(0 \%)\end{array}$ \\
\hline
\end{tabular}

* ANOVA analysis found a significant difference between the BVFI group and the other two groups in terms of the proportion who were premature $(p<0.046)$.

\# Fisher Exact Test found a significant difference in decannulation rates between groups $(\mathrm{p}=0.001)$.

\#\#Fisher Exact Test found a significant difference in decannulation rates between groups $(\mathrm{p}=0.002)$. 
Figure Legend:

Figure 1. (a-b) Preoperative and (c-d)post-operative photos of patient with posterior glottic stenosis.

Figure 2. Decannulation rates after endoscopic and open grafting. There was a statistically significant difference between groups based upon indication for surgery, $(p=0.002)$.

Figure 3. Decannulation rates after endoscopic grafting. There was a statistically significant difference between groups based upon indication for surgery, $(p=0.001)$. Figure 4. Treatment Algorithm 